Journal of Patient-Centered

Volume 7

Issue 4 -- Women and Cancer

Article 7

$10-23-2020$

\title{
Discordance in Perceptions of Barriers to Breast Cancer Treatment Between Hispanic Women and Their Providers
}

\author{
Swapna Reddy \\ Mary Saxon \\ Nina Patel \\ Matthew Speer \\ Tiffany Ziegler \\ Nirali Patel \\ Madison Ziegler \\ Stephany Esquivel \\ Andrea Daniella Mata \\ Asha Devineni \\ See next page for additional authors \\ Follow this and additional works at: https://aah.org/jpcrr \\ Part of the Community-Based Research Commons, Community Health Commons, Community Health \\ and Preventive Medicine Commons, Health Services Research Commons, Medicine and Health \\ Commons, Oncology Commons, Race and Ethnicity Commons, and the Women's Health Commons
}

\section{Recommended Citation}

Reddy S, Saxon M, Patel N, Speer M, Ziegler T, Patel N, Ziegler M, Esquivel S, Mata AD, Devineni A, Paode P, Thawani N, Mutyala S. Discordance in perceptions of barriers to breast cancer treatment between Hispanic women and their providers. J Patient Cent Res Rev. 2020;7:337-42. doi: 10.17294/

2330-0698.1751

Published quarterly by Midwest-based health system Advocate Aurora Health and indexed in PubMed Central, the Journal of Patient-Centered Research and Reviews (JPCRR) is an open access, peer-reviewed medical journal focused on disseminating scholarly works devoted to improving patient-centered care practices, health outcomes, and the patient experience. 


\section{Discordance in Perceptions of Barriers to Breast Cancer Treatment Between Hispanic Women and Their Providers}

\section{Authors}

Swapna Reddy, Mary Saxon, Nina Patel, Matthew Speer, Tiffany Ziegler, Nirali Patel, Madison Ziegler, Stephany Esquivel, Andrea Daniella Mata, Asha Devineni, Pooja Paode, Nitika Thawani, and Subhakar Mutyala 


\title{
Discordance in Perceptions of Barriers to Breast Cancer Treatment Between Hispanic Women and Their Providers
}

\author{
Swapna Reddy, JD, DrPH, ${ }^{1}$ Mary Saxon, MS, ${ }^{1}$ Nina Patel, MS, ${ }^{1}$ Matthew Speer, MS, ${ }^{1}$ Tiffany \\ Ziegler, MS, ${ }^{2}$ Nirali Patel, MS, ${ }^{3}$ Madison Ziegler, ${ }^{1}$ Stephany Esquivel, MS, ${ }^{1}$ Andrea Daniella Mata, ${ }^{4}$ \\ Asha Devineni, ${ }^{5}$ Pooja Paode, MPP, MS, ${ }^{1}$ Nitika Thawani, MD, ${ }^{6}$ Subhakar Mutyala, MD, MPH ${ }^{6}$ \\ ${ }^{1}$ College of Health Solutions, Arizona State University, Phoenix, AZ; ${ }^{2}$ Arizona College of Osteopathic Medicine, \\ Midwestern University, Glendale, AZ; ${ }^{3}$ David Geffen School of Medicine at UCLA, Los Angeles, CA; ${ }^{4}$ College of Liberal \\ Arts, University of Texas at El Paso, El Paso, TX; ${ }^{5}$ Sandra Day O'Connor College of Law, Arizona State University, \\ Phoenix, AZ; ${ }^{6}$ University of Arizona College of Medicine - Phoenix, Phoenix, AZ
}

\begin{abstract}
Despite comparable screening and incidence rates that are $26 \%$ below that of non-Hispanic Whites, Hispanic women present with breast cancer at more advanced stages of disease, representing a continuing and troubling health disparity for this population. Reducing these disparities warrant more innovative research approaches to better understand perspectives of Hispanic patients regarding barriers to treatment and how these perspectives compare to those of their providers. A pilot qualitative study was conducted at a major urban cancer center in Arizona that measured both patient and provider perspectives regarding barriers to treatment. Through a multimethod qualitative analysis, researchers surveyed patients and providers to identify perceived barriers and discordance in shared understanding. Data collection and analysis consisted of surveying patients and providers, then performing inductive qualitative analysis. Results indicated the highest concordance, or shared understanding, between patients and providers was in recognizing barriers within delivery of care, such as cost of care and insurance coverage. The greatest discordance, or gaps in shared understanding, existed in upstream barriers of the health care system, such as emotional support and trust in systems. These results underscore the gap in shared understanding between patients and providers regarding upstream barriers to care as well as the nonclinical social determinants of health Hispanic patients face in accessing breast cancer treatment. More research is warranted using this approach as a tool to reduce health disparities. (J Patient Cent Res Rev. 2020;7:337-342.)
\end{abstract}

Keywords breast cancer; health disparities; Hispanic women; social determinants of health; provider-patient relationship; health equity; patient focus

$\mathrm{H}$ ispanics are the largest and fastest-growing minority population in the United States and are projected to make up almost $30 \%$ of the national population by 2060 . Even with this rapid growth, Hispanics are still overrepresented among socioeconomic factors such as poverty and health disparities. ${ }^{1}$ A key health disparity example is that of breast cancer, which is the leading cause of cancer death among Hispanic women in the United States and

Corresponding author: Swapna Reddy, JD, DrPH, College of Health Solutions, Arizona State University, Health North Suite 501, PO Box 879020,

Tempe, AZ 85287-9020 (swapna.reddy@asu.edu) the second leading cause of cancer deaths among U.S. women at large. ${ }^{2-4}$ Despite incidence rates that can be $26 \%$ below that of non-Hispanic Whites and similar screening rates, Hispanic women are at higher risk for early-onset breast cancer, more advanced stage, and more aggressive tumor types as compared with nonHispanic White women. ${ }^{5-7}$ Differences in presentation of disease, despite lower incidence, represent a continuing and troubling health disparity for this population.

Disparities are largely due to delays in treatment and reduced likelihood for completion of recommended protocols. ${ }^{8,9}$ Understanding the causes of these delays is critical to reducing these disparities. Often, factors that lead to treatment delays in vulnerable populations are rooted in their social determinants of health. ${ }^{10-12}$ Recognizing these 
root causes highlights the need for a shared understanding of the lived experiences of the patients in this demographic and the providers that serve their community.

Closing gaps in breast cancer survival rates requires crafting interventions that are responsive to the needs of the target population by understanding Hispanic patients' perspective and the upstream factors that could be potentially impacting treatment decisions. Understanding these factors necessitates an acknowledgment that the target population is often experiencing a parallel continuum of social determinants of health that exacerbate disparities in breast cancer outcomes.

Health providers are important stakeholders in our health care delivery system and key influencers in patient care, therefore they must understand the barriers patients face in treatment. To date, there has been minimal research comparing perceptions of barriers to cancer care and cancer treatment between patients of minority race/ ethnicity, especially Hispanic women, with those of their health care team. Discordance between providers and their patients exacerbates disparities and can thwart effective, timely, patient-centered treatment. ${ }^{13,14}$

We seek to close this gap in the literature through a pilot study assessing the perceptions of providers and patients related to barriers to care and treatment faced by Hispanic female patients with breast cancer from a single institution in the U.S. Southwest.

\section{METHODS}

This was a multimethod qualitative study performed as a multidisciplinary collaboration between health services faculty and researchers from a large public university and health care providers from a major urban academic cancer center, both located in Phoenix, Arizona. After careful synthesis of the existing literature, we designed and administered semi-structured interview scripts for patients and survey instruments for providers to assess perceptions of the barriers faced by Hispanic women in accessing breast cancer treatment.

To protect human subjects, the study was submitted to and approved by the institutional review board committee of the research university and treating hospital.

\section{Patient Interviews}

The initial protocol included in-person focus groups with patients. However, due to feasibility and attendance concerns, the protocol was adjusted for the interviews to be conducted via phone calls. The patient interviews were semi-structured and conducted via phone calls by 2 bilingual members of the research team (S.E. and A.D.M.) and were recorded using voice memos (Table 1). Interviews took place between May 2018 and July 2018 and were limited to 30 minutes for each participant. These patient interviews were utilized to identify the actual barriers to breast cancer treatment that are experienced by the patient population. Patients consisted of 14 female self-identified Hispanic patients who had recently received or were currently receiving breast cancer treatment at a major urban cancer center in Phoenix.

\section{Provider Surveys}

Provider surveys consisted of a combination of Likert scale and open-ended questions administered via online questionnaire (Online Appendix A). Surveys

Table 1. Patient Interview Questions

1. At this point in time, have you completed your treatment? Do you mind me asking how you found out about your diagnosis, were you screened, annual? Noticed yourself?

2. Do you think accessing the care you need is difficult?

3. If YES, what do you think makes getting care you need so difficult?

4. What are the barriers you face in accessing the health care treatment you need? Again, this could include insurance, finances, work, transportation, etc.

5. What is the biggest thing you need help with throughout this process?

6. Did you feel comfortable with your treatment plan?

7. Do you understand your treatment plan?

8. Do you feel comfortable with your health care providers? Why or why not?

9. What issues arose when you are trying to seek care for your breast cancer?

10. What kind of social support system do you have? Family, friends?

11. Did you work? If YES, how has that helped/hindered you in this process?

12. Do you have any children? If YES, what arrangements do you have for child care?

13. Is there anything else you would like to share before concluding the survey? 
were collaboratively designed with cancer center staff, clinicians, and researchers to assess provider perceptions of their patients' barriers to treatment. The providers surveyed consisted of physicians and other allied health professionals who were involved in the care continuum for patients with breast cancer at the cancer center. Participants were recruited through an existing academic partnership with the cancer center and a network of "clinical champions" - practicing clinicians involved with the study design. Responses were collected from June 2017 through October 2017.

Institutional review board approval was granted for the semi-structured patient interviews and for the provider surveys through both institutions.

\section{Qualitative Analysis}

Interviews and surveys were analyzed utilizing qualitative data analysis methods based on grounded theory, including coding and memoing, to identify themes. ${ }^{15}$ These themes and inclusion/exclusion criteria were defined in the codebook, which multiple coders used to triangulate interpretations of data and ensure intercoder reliability. ${ }^{16}$ Analysis of the qualitative data involved coding responses from patient interviews and provider surveys to identify meta-themes that influence breast cancer health decisions. A Kappa test was then performed by the two participating researchers to remove coder bias and further confirm that each barrier had been sufficiently recorded and sorted into appropriately corresponding categories.

The Kappa test included each researcher separately analyzing the transcripts, referencing the original codebook, appropriately identifying barriers, and confirming that a properly suited barrier code was created as per the codebook definitions and inclusion/exclusion criteria. Any discrepancies in barrier codes in transcripts were expelled by redefining, adjusting, or adding to the barrier code definition, inclusion, and exclusion criteria.

Coding was repeated for provider surveys. Prevalence of themes in provider and patient responses was compared to identify areas of concordance and discordance.

\section{RESULTS}

In total, there were 12 themes sorted into 4 main categories (financial constraints, social factors, barriers in health care delivery, accessibility constraints) as displayed in the codebook (Online Appendix B). Definitions for each of these themes and inclusion/exclusion criteria were revised and finalized in the codebook to adequately reflect the barriers.

The number of patients who responded was 14 , and the number of providers who responded was 23. Providers consisted of the following physicians and other allied health professionals: 4 radiation oncologists, 1 surgical oncologist, 1 medical oncologist, 6 nurses, 4 medical assistants, 1 licensed clinical social worker, 1 patient advocate, 1 American Cancer Society patient navigator, 1 genetic counselor, 2 radiation therapists, and 1 Spanish medical interpreter.

Table 2 compares the barriers coded from patient interviews and provider surveys, in decreasing order of prevalence. Barriers are color-coded to their corresponding domain:

Table 2. Top Responses for Barriers to Health

\begin{tabular}{l|l}
\hline Top patient responses $(\mathbf{n}=14)$ & Top provider responses $(\mathbf{n}=\mathbf{2 3})$ \\
\hline 1. Employment Conflicts $(8$ of $14,57 \%)$ & 1. Cost of Care $(14$ of $23,61 \%)$ \\
\hline 2. Emotional Support $(5$ of $14,36 \%)$ & 2. Insurance Coverage $(12$ of $23,52 \%)$ \\
\hline 2. Cost of Care $(5$ of $14,36 \%)$ & 3. Care Coordination $(9$ of $23,39 \%)$ \\
\hline 3. Trust in Health Systems $(4$ of $14,29 \%)$ & 4. Health Literacy $(8$ of $23,35 \%)$ \\
\hline 3. Care Coordination $(4$ of $14,29 \%)$ & 5. Language Limitations $(6$ of $23,26 \%)$ \\
\hline 3. Health Literacy $(4$ of $14,29 \%)$ & 6. Transportation $(5$ of $23,22 \%)$ \\
\hline 4. Insurance Coverage $(3$ of $14,21 \%)$ & 7. Emotional Support $(4$ of $23,17 \%)$ \\
\hline 4. Language Limitations $(3$ of $14,21 \%)$ & 8. Cultural Views $(3$ of $23,13 \%)$ \\
\hline 5. Childcare Needed $(2$ of $14,14 \%)$ & 9. Childcare Needed $(1$ of $23,4 \%)$ \\
\hline 5. Transportation $(2$ of $14,14 \%)$ & 9. Employment Conflicts $(1$ of $23,4 \%)$ \\
\hline 5. Immigration Status $(2$ of $14,14 \%)$ & 9. Immigration Status $(1$ of $23,4 \%)$ \\
\hline 6. Cultural Views $(1$ of $14,7 \%)$ & 10. Trust in Health Systems $(0$ of $23,0 \%)$ \\
\hline
\end{tabular}




\section{Financial Constraints:}

\section{Insurance coverage}

One patient explained, "the insurance did not allow them to do exams that weren't on the record."

\section{Cost of care}

Patients often reported a combination of the high out-of-pocket costs of treatment paired along with their inability to pay due to lack of insurance coverage, inability to work, and transportation issues. As described by one patient, "I had to find other options because there wasn't - I didn't have a way [of] obtaining that treatment. I looked in many places.... Yes, it was difficult."

\section{Immigration/Employment status}

One patient explained, "I don't have immigration status, and I qualified for many grant aids, [but] I also couldn't access that help because I am neither a resident or a citizen."

\section{Social Factors:}

\section{Emotional support}

One woman explained the impact of limited social support by stating that her first appointment was difficult without her "family with her that would give her more courage."

\section{Cultural views toward cancer and treatment}

One woman described her cultural views toward cancer treatment by explaining, "for the Hispanic culture we are hard on ourselves because we used to be so independent ... and not someone who wants to get the help or ask for help.... In the Hispanic culture you don't get to be outspoken ... it's more saying she got cancer and maybe this is what God wanted for her."

\section{Trust in health systems}

One patient described a case in which she felt that her health providers "didn't believe [her], they wanted to do things their way ... they wanted to treat [her] for other illnesses."

\section{Barriers in Health Care Delivery:}

\section{Care coordination}

As expressed by the patients, there is a struggle in navigating the health care system. For instance, one patient stated that there is a need for "a link or a person that guides [you], a person that tells [you] what are your options and where you can go to continue to receive treatment or where [you] can find help."

\section{Health literacy}

The results from the patient interviews indicated that inadequate health literacy presented barriers when accessing care. In fact, one patient noted that a significant barrier was not feeling knowledgeable about the side effects and what occurs after diagnosis, that "[you] have to find [the information] out on [your] own or ask someone who has already gone through it," as the health care system does not adequately cater to this struggle. Other patients stated the need for more information regarding treatment options and expectations throughout the treatment process.

\section{Language limitations}

Several patients revealed that there were gaps in communication between the provider and patient, often with a shortage of interpreters on staff. One particular woman explained, "few [of the] medics that can answer you directly, give you information directly; we have to recruit interpreters."

\section{Accessibility Constraints:}

Childcare accessibility

One patient states, "I did have to make a lot of adjustments when it came to it because my kids have to go to school ... the days that I was tired I would have to have help at home ... it was all hard, really hard."

\section{Employment conflicts}

One patient stated that she "had to leave [work] due to treatment because [she] did not have time for both."

\section{Transportation}

Often, patients indicated that they had difficulty finding affordable or accessible means of transportation to their health appointments. One of the interviewed patients explained, "I didn't have a car. I didn't have strength. I was very weak. I was very fatigued." 
financial constraints (yellow shades), social factors (green), barriers in health care delivery (blue), and accessibility constraints (red). Table 3 provides several of the most relevant patient quotes pertaining to each barrier.

\section{DISCUSSION}

Our results indicate discordance in perspectives between patients and providers regarding Hispanic women's barriers to breast cancer treatment. The discordance in this small cohort may represent a gap in shared understanding between those who treat relative to the challenges of those being treated. The smallest gaps in concordance were observed in determinants that providers regularly interface with inside the clinical walls of the health care system, such as cost of care or care coordination. Larger gaps in discordance exist among the barriers more likely to be experienced by patients outside of the clinical walls, such as employment conflicts, emotional support, or trust in health systems. These gaps suggest providers lack understanding of their patients' lived experiences beyond direct interaction with the health system. Further investigation into the perceptions of patients and providers is warranted.

While immigration status was not a top barrier cited by patients, we found it significant to discuss in light of this topic's focus at the state and national levels and its likely impact on Hispanic patients. The issue of status is important in the context of treatment because undocumented immigrants are ineligible for federal health insurance programs. ${ }^{17}$ Fear of discovery and deportation leads many unauthorized immigrants to delay treatment of serious acute conditions and chronic illnesses. ${ }^{18,19}$ Lack of clarity on immigration policies and relative enforcement, such as the now halted "public charge rule," enables a system of fear and uncertainty. Previous studies have supported the strong role of immigration concerns in Hispanic women's health decisions. ${ }^{19}$ We suspect that this climate of fear has led to a drastically high underreporting rate of this barrier by patients.

\section{Study Limitations}

This research was a multimethod snapshot study with a small sample size in both patients and providers; future studies should note its limitations. A small sample size may impact the generalizability of findings; however, in qualitative studies, saturation of responses is more important to the validity of a study than sample size. ${ }^{20}$ Potential selection bias exists as all study participants either receive treatment or provide care at the same large urban institution. As patients received care in a tertiary cancer center, the data might be skewed toward patients with insurance, transportation, and health literacy. Additionally, as all participants interviewed were former or current patients, they exhibited the ability to overcome barriers and access treatment at least minimally. As such, the study was not able to capture the experiences of those women whose barriers were too significant to receive treatment at all.

The stage of the patient's breast cancer and the time they underwent treatment were not captured. Both of these factors could influence the patient's perceptions of the health care system as it relates to their own experiences, needs, and barriers encountered and could therefore influence their answers. Similarly, the providers were a mix of physicians, nurses, and allied health professionals. Perceptions of patients' barriers could vary across these different professions. As the sample size was small, the data could not be stratified based on the patient's stage, length of treatment, or the respondents' profession. Further study into these differences is warranted.

This study suggests a discordance between patients and their health care providers, specifically regarding upstream and social determinants of health experienced by Hispanic female patients with breast cancer. The discordance observed in the study is suggestive of a lack of shared understanding between providers toward the lived experiences and barriers of their patients. Though the findings are notable as they pertain to breast cancer disparities, this pilot snapshot represents an innovative approach that underscores the need to think more about the role of shared understanding between cancer patients and providers regarding perceived barriers as a tool to reduce health disparities. Additional studies utilizing this approach in different contexts are warranted.

\section{Patient-Friendly Recap}

- Hispanic women in the United States are diagnosed with breast cancer less frequently than non-Hispanic White women but, on average, with more advanced disease. This disparity may be due to individual, social, economic, and systemic differences, including factors specific to the health system and care provided.

- The authors interviewed both health care providers and Hispanic women with breast cancer treated at the same institution to obtain each's perspective on possible barriers to treatment.

- They found that the greatest gaps in shared understanding between patients and providers involved barriers patients face outside the health care system, such as employment conflicts or a lack of emotional support. 


\section{Author Contributions}

Study design: Reddy, Mutyala. Data acquisition or analysis: Saxon, Nina Patel, T. Ziegler, Nirali Patel, M. Ziegler, Esquival, Mata, Devineni, Paode. Manuscript drafting: Reddy, Saxon, Nina Patel, Speer, M. Ziegler, Mutyala. Critical revision: Reddy, Saxon, Nina Patel, Speer.

\section{Conflicts of Interest}

None.

\section{Funding Sources}

Andrea Daniella Mata was supported by a grant from the National Institutes of Health under linked award numbers RL5GM118969, TL4GM118971, and UL1GM118970.

\section{References}

1. Edwards A; U.S. Census Bureau. Hispanic poverty rate hit an all-time low in 2017. Rate dropped to 18.3 percent, down 1.1 points from 2016. Published February 27, 2019; accessed April 21, 2020. https://www.census.gov/library/stories/2019/02/ hispanic-poverty-rate-hit-an-all-time-low-in-2017.html

2. U.S. Census Bureau. QuickFacts: United States. Accessed April 21, 2020. https://www.census.gov/quickfacts/fact/table/ $\underline{\mathrm{US} / \mathrm{RHI} 725218}$

3. U.S. Census Bureau. Hispanic population to reach 111 million by 2060. Last revised October 4, 2018; accessed April 21, 2020. https://www.census.gov/library/visualizations/2018/ comm/hispanic-projected-pop.html

4. Nahleh Z, Otoukesh S, Mirshahidi HR, et al. Disparities in breast cancer: a multi-institutional comparative analysis focusing on American Hispanics. Cancer Med. 2018;7:2710-7. Crossref

5. National Center for Health Statistics; Centers for Disease Control and Prevention. Health, United States, 2018-Data Finder - Use of mammography among women aged 40 and over, by selected characteristics: United States, selected years 1987-2015 (Table 33). Accessed April 21, 2020. https://www. cdc.gov/nchs/data/hus/2018/033.pdf

6. Siegel RL, Fedewa SA, Miller SD, et al. Cancer statistics for Hispanics/Latinos, 2015. CA Cancer J Clin. 2015;65:457-80. Crossref

7. Yedjou CG, Tchounwou PB, Payton M, et al. Assessing the racial and ethnic disparities in breast cancer mortality in the United States. Int J Environ Res Public Health. 2017;14(5):486. $\underline{\text { Crossref }}$
8. American Cancer Society. Cancer Facts \& Figures for Hispanics/Latinos, 2018-2020. American Cancer Society, Inc.; 2018.

9. Power EJ, Chin ML, Haq MM. Breast cancer incidence and risk reduction in the Hispanic population. Cureus. 2018;10(2):e2235. Crossref

10. Nonzee NJ, Ragas DM, Ha Luu T, et al. Delays in cancer care among low-income minorities despite access. $J$ Womens Health (Larchmt). 2015;24:506-14. Crossref

11. Mandelzweig L, Goldbourt U, Boyko V, Tanne D. Perceptual, social, and behavioral factors associated with delays in seeking medical care in patients with symptoms of acute stroke. Stroke. 2006;37:1248-53. Crossref

12. Sterling S, Chi F, Weisner C, et al. Association of behavioral health factors and social determinants of health with high and persistently high healthcare costs. Prev Med Rep. 2018;11:154-9. $\underline{\text { Crossref }}$

13. Rice LJ, Jefferson M, Melvin CL, Halbert $\mathrm{CH}$. Concordance in patient and provider priorities and preferences to address behavioral risk factors for cancer health disparities interventions. (abstr.) Cancer Epidemiol Biomarkers Prev. 2016;25(3 Suppl):abstract nr A08. Crossref

14. Albarracín D, Johnson BT (eds). The Handbook of Attitudes Volume 2: Applications, 2nd Edition. Routledge; 2019.

15. Corbin J, Strauss A. Basics of Qualitative Research: Techniques and Procedures for Developing Grounded Theory, 3rd Edition. Sage Publications, Inc.; 2008. Crossref

16. Kurasaki KS. Intercoder reliability for validating conclusions drawn from open-ended interview data. Field Methods. 2000;12:179-94. Crossref

17. National Conference of State Legislatures. Immigrant eligibility for health care programs in the United States. Published October 19, 2017; accessed April 21, 2020. http:// www.ncsl.org/research/immigration/immigrant-eligibility-forhealth-care-programs-in-the-united-states.aspx

18. Nyblade L, Stockton M, Travasso S, Krishnan S. A qualitative exploration of cervical and breast cancer stigma in Karnataka, India. BMC Womens Health. 2017;17(1):58. Crossref

19. Heyman JM, Núñez GG, Talavera V. Healthcare access and barriers for unauthorized immigrants in El Paso County, Texas. Fam Community Health. 2009;32:4-21. Crossref

20. Vasileiou K, Barnett J, Thorpe S, Young T. Characterising and justifying sample size sufficiency in interview-based studies: systematic analysis of qualitative health research over a 15-year period. BMC Med Res Methodol. 2018;18(1):148. Crossref

(C) 2020 Advocate Aurora Health, Inc. 